\title{
Finite element modeling of macrosegregation coupled with shrinkage cavity in steel ingots using arbitrary Lagrangian-Eulerian model
}

\author{
Kang-xin Chen, Hao Shi, and *Hou-fa Shen \\ Key Laboratory for Advanced Materials Processing Technology, Ministry of Education; School of Materials Science and Engineering, \\ Tsinghua University, Beijing 100084, China
}

\begin{abstract}
Shrinkage cavity has significant influence on macrosegregation in steel ingots. An arbitrary Lagrangian-Eulerian (ALE) model based on volume averaging method is developed to predict the coupled formation progress of macrosegregation and shrinkage cavity during solidification of steel ingots. The combined effect of thermal-solutal convection and solidification shrinkage on macrosegregation is considered in the model. A specially designed mesh update algorithm is proposed to consider the formation of shrinkage cavity. The streamline-upwind/Petrov-Galerkin (SUPG) stabilized finite element algorithm is adopted to solve the conservation equations. Two solution methods for the energy conservation equation are proposed, i.e. the temperature-based solver and enthalpy-based solver. A Pb-48wt.\%Sn solidification benchmark is used for validation. Then, the ALE model is applied to a Fe-3.6wt.\%C industrial steel ingot. The formation progress of macrosegregation coupled with shrinkage cavity is predicted. By comparison with the predictions of the finite element model and finite volume model, the effect of shrinkage cavity formation on macrosegregation is investigated. Results show that the formation of shrinkage cavity can significantly change the segregation region and segregation degree at the hot top. It is demonstrated that the ALE model can predict the coupled formation of macrosegregation and shrinkage cavity in steel ingots.
\end{abstract}

Key words: steel ingot; macrosegregation; shrinkage cavity; ALE model; finite element modeling CLC numbers: TG142.1/TP391.9Ｄocument code: A Article ID: 1672-6421(2019)05-291-09

$\mathrm{M}$ acrosegregation, i.e. composition heterogeneity at the macroscopic scale, is a serious defect for steel ingots. The macrosegregation defect forms during solidification and is difficult to eliminate in subsequent processes. Macrosegregation results from the relative motion of the solute-depleted solid phase and soluteenriched liquid phase ${ }^{[1,2]}$. During the solidification of steel ingots, a large shrinkage cavity forms at the top due to solidification shrinkage. Experimental studies show that the formation of a shrinkage cavity has a strong impact on macrosegregation in large steel ingots ${ }^{[3]}$.

Considerable effort has been devoted to model development for macrosegregation and shrinkage cavity. However, studies focusing on the coupled prediction of macrosegregation and shrinkage cavity are limited. Some studies examine the effect of solidification shrinkage on

\section{*Hou-fa Shen}

Male, born in 1964, Ph.D., Professor. Research interests: modeling and simulation of solidification process related to casting.

E-mail: shen@tsinghua.edu.cn

Received: 2019-05-08; Accepted: 2019-07-06 macrosegregation in the absence of a shrinkage cavity ${ }^{[4-7]}$. In these studies, an open boundary is provided or a flat free surface is assumed for the ingot riser. The simulation results show that the effect of solidification shrinkage is negligible except at the chill wall where inverse segregation forms. As for steel ingots, however, only a few studies consider the coupling of shrinkage cavity and macrosegregation. Zhang et al. ${ }^{[8,9]}$ developed a single-domain multi-phase model by using a volume of fluid (VOF) method to track the interface between the metal and air. The interchange of liquid between the part and riser was emphasized to study the influence of riser configuration on macrosegregation. The formation of shrinkage cavity was shown to increase the average composition of the part. Wang et al. ${ }^{[10,11]}$ developed a three-phase solidification model to simulate the solidification progress of $\mathrm{Al}-4 \mathrm{wt} . \% \mathrm{Cu}$ alloy, and the air phase was added to track the free surface. Completely different macrosegregation maps were obtained with and without considering the shrinkage feeding flow. Wu et al. ${ }^{[12,13]}$ extended a previous three-phase mixed columnar-equiaxed solidification model by adding 
additional gas (or covering slag) phase to treat the formation of a shrinkage cavity; then the four-phase model was applied on a $2.45 \mathrm{t}$ ingot and a $10.5 \mathrm{t}$ ingot. Compared with the previous threephase model, the agreement between the new four-phase model and the experiment was obviously improved, demonstrating the importance and necessity to consider the effect of a shrinkage cavity.

During the past two years, Ge et al. ${ }^{[14,15]}$ developed a fourphase dendritic solidification model, similar with the model developed by $\mathrm{Wu}$ et al. ${ }^{[13]}$, to further consider the dendritic structure of the equiaxed grains, and the model was applied to a $55 \mathrm{t} \mathrm{Fe}-3.3 \mathrm{wt} . \% \mathrm{C}$ steel ingot and an $\mathrm{Al}-4.5 \mathrm{wt} . \% \mathrm{Cu}$ ingot. The simulation results showed that the shrinkage reduced the range and changed the location of positive segregation at the hot top of the ingot. In the above studies, fixed mesh methods, such as the VOF method or the gas-phase-included multiphase model method, were adopted to predict the shrinkage cavity. The moving mesh method, or arbitrary Lagrangian-Eulerian (ALE) method, can also be used for free surface problems. Compared with fixed mesh methods, the ALE method provides a more accurate description of the free surface ${ }^{[16]}$. Bellet and coworkers $[16,17]$ introduced this method into the solidification and casting research area to simulate the mold filling process and shrinkage cavity formation. It was verified that the free surface during the mold filling and solidification progresses could be properly solved by an ALE method. In a previous study ${ }^{[7]}$, the authors developed an ALE model to predict macrosegregation caused by the thermal-solutal convection and solidification shrinkage in a $\mathrm{Pb}-19.2 \mathrm{wt}$ \% $\mathrm{Sn}$ test casting. Inverse segregation was predicted at the chill wall and it was demonstrated that solidification shrinkage delayed the advance of the solidification front and intensified the segregation. However, a flat free surface was assumed in the $\mathrm{Pb}-19.2 \mathrm{wt} . \% \mathrm{Sn}$ casting, thus the shape of shrinkage cavity was simplified.

In this study, a volume-averaged ALE model, considering both of the thermal-solutal convection and solidification shrinkage, is developed to predict macrosegregation coupled with shrinkage cavity in steel ingots. First, the governing equations of the ALE model are deduced based on volume averaging method. Afterwards, the finite element algorithm for the ALE model is introduced. Then, a Pb-48wt.\% Sn solidification benchmark is used for validation. Finally, the ALE model is applied to a 3.3 ton industrial steel ingot to predict macrosegregation coupled with shrinkage cavity.

\section{Mathematical model and numerical algorithm}

Different from the ALE model previously developed based on mixture theory ${ }^{[7]}$, the ALE model in this study is developed based on volume averaging method, which is more commonly used for model derivation.

\subsection{ALE model}

The volume-averaged model considering solidification shrinkage is developed based on the following assumptions:

(1) The melt flow is incompressible and Newtonian.

(2) The solid motion is neglected; thus, neither grains sedimentation nor mushy zone deformation is considered.

(3) The mushy zone is modeled as an isotropic porous medium saturated with liquid, and the permeability is defined by the Carman-Kozeny formula.

(4) The densities of the solid and liquid phases are different albeit constant, and the Boussinesq approximation is used in the buoyancy term of the momentum conservation equation.

(5) The thermal-physical properties of the solid and liquid phases are different albeit constant.

(6) The lever rule is used to describe microsegregation.

(7) No internal shrinkage porosity is considered during solidification; thus, the casting is sound during solidification.

The conservation equations of the volume-averaged model considering solidification shrinkage have been derived in the Eulerian formulations ${ }^{[5]}$, which are summarized as follows:

Mass conservation:

$$
\nabla \cdot \boldsymbol{u}=-\frac{1}{\rho_{1}} \frac{\partial \bar{\rho}}{\partial t}
$$

where $\boldsymbol{u}$ is the superficial velocity, denoted by $\boldsymbol{u}=g_{1} \boldsymbol{u}_{1}$, with $\boldsymbol{u}_{1}$ being the volume-averaged intrinsic velocity of the liquid phase; $\bar{\rho}$ is the mixture density, which is volume-averaged as $\bar{\rho}=g_{\mathrm{s}} \rho_{\mathrm{s}}+g_{1} \rho_{1} ; g_{\mathrm{s}}$ and $g_{1}$ denote the volume fractions of the solid and liquid phases; $\rho_{\mathrm{s}}$ and $\rho_{1}$ denote the densities for the solid and liquid phases; $t$ is the time.

Momentum conservation:

$$
\frac{\partial \boldsymbol{u}}{\partial t}+\frac{\boldsymbol{u}}{g_{1}} \cdot \nabla \boldsymbol{u}+\frac{\boldsymbol{u}}{g_{1}} \nabla \cdot \boldsymbol{u}=-\frac{g_{1}}{\rho_{1}} \nabla P+\frac{\mu_{1}}{\rho_{1}} \nabla \cdot \nabla \boldsymbol{u}-\frac{\mu_{1}}{\rho_{1}} \frac{g_{1}}{K} \boldsymbol{u}+g_{1} \frac{\rho_{1}^{\mathrm{b}}}{\rho_{1}} \boldsymbol{g}
$$

where $P$ is the intrinsic pressure in the liquid phase, $\mu_{1}$ denotes the dynamic viscosity of the liquid phase, $g$ is the gravity vector, $K$ denotes the permeability defined by the Carman-Kozeny model, $\rho_{1}^{\mathrm{b}}$ denotes the liquid density in the buoyancy term. $K$ and $\rho_{1}^{\mathrm{b}}$ are given as follows:

$$
\begin{gathered}
K=\lambda_{2}^{2} g_{1}^{3} / g_{\mathrm{s}}^{2} / 180 \\
\rho_{1}^{\mathrm{b}}=\rho_{1}\left[1-\beta_{\mathrm{T}}\left(T-T_{\text {ref }}\right)+\beta_{\mathrm{w}}\left(w_{1}-w_{\text {ref }}\right)\right]
\end{gathered}
$$

where $\lambda_{2}$ denotes the secondary dendrite arm spacing, $\beta_{\mathrm{T}}$ denotes the thermal expansion coefficient, $\beta_{\mathrm{w}}$ denotes the solutal expansion coefficient, $T_{\text {ref }}$ and $w_{\text {ref }}$ denote the reference temperature and solute concentration.

Energy conservation:

$$
\bar{\rho} \frac{\partial H}{\partial t}+\rho_{1} \boldsymbol{u} \cdot \nabla H_{1}=\nabla \cdot(k \nabla T)+\left(H-H_{1}\right) \rho_{1} \nabla \cdot \boldsymbol{u}
$$

where $H$ is the mass-averaged enthalpy, which is given by $H=f_{\mathrm{s}} H_{\mathrm{s}}+f_{1} H_{1} ; H_{\mathrm{s}}$ and $H_{1}$ denote the enthalpies in the solid and liquid phases; $f_{\mathrm{s}}$ and $f_{1}$ denote the mass fractions of the solid and liquid phases; $k$ is the volume-averaged thermal conductivity, which is given by $k=g_{\mathrm{s}} k_{\mathrm{s}}+g_{1} k_{\mathrm{l}}$. 
Solute conservation:

$$
\bar{\rho} \frac{\partial w}{\partial t}+\rho_{1} \boldsymbol{u} \cdot \nabla w_{1}=\rho_{1} D_{1} \nabla \cdot\left(g_{1} \nabla w_{1}\right)+\left(w-w_{1}\right) \rho_{1} \nabla \cdot \boldsymbol{u}
$$

where $w$ is the mass-averaged mass fraction of solute, which is defined as $w=f_{\mathrm{s}} w_{\mathrm{s}}+f_{1} w_{1} ; w_{\mathrm{s}}$ and $w_{1}$ denote the mass fractions of solute in the solid and liquid phases; $D_{1}$ is the diffusion coefficient in the liquid phase.

In this study, the ALE method is adopted to predict the free surface evolution and the formation progress of a shrinkage cavity during solidification of steel ingots. Thus, the ALE formulations are adapted from the Eulerian formulations. Except for the different coordinate systems ( $x$ denotes the Eulerian spatial system, $\chi$ denotes the ALE spatial system) between the ALE form and Eulerian form, the ALE formulations and Eulerian formulations are similar in form. When modified from the Eulerian form to ALE form, the only modification is that all materials time derivatives are replaced by the ALE form of material time derivatives ${ }^{[18]}$. Considering a specific function $f$, the term $\partial f / \partial t$ in the Eulerian form will be replaced by $\partial f / \partial t$ $\boldsymbol{u}_{\text {mesh }} \nabla f$ in the ALE form, where $\boldsymbol{u}_{\text {mesh }}$ is the mesh velocity. Details about the ALE theory can be found in the references ${ }^{[16,18]}$. Here, the ALE formulations of the model are summarized as follows:

$$
\begin{gathered}
\nabla \cdot \boldsymbol{u}(\chi, t)=-\frac{1}{\rho_{1}}\left[\frac{\partial \bar{\rho}(\chi, t)}{\partial t}-\boldsymbol{u}_{\text {mesh }} \cdot \nabla \bar{\rho}(\chi, t)\right] \\
\frac{\partial \boldsymbol{u}(\chi, t)}{\partial t}-\boldsymbol{u}_{\mathrm{mesh}} \cdot \nabla \boldsymbol{u}(\chi, t)+\frac{\boldsymbol{u}(\chi, t)}{g_{1}} \cdot \nabla \boldsymbol{u}(\chi, t)+\frac{\boldsymbol{u}(\chi, t)}{g_{1}} \nabla \cdot \boldsymbol{u}(\chi, t) \\
=-\frac{g_{1}}{\rho_{1}} \nabla P(\chi, t)+\frac{\mu_{1}}{\rho_{1}} \nabla \cdot \nabla \boldsymbol{u}(\chi, t)-\frac{\mu_{1}}{\rho_{1}} \frac{g_{1}}{K} \boldsymbol{u}(\chi, t)+g_{1} \frac{\rho_{1}^{\mathrm{b}}}{\rho_{1}} \boldsymbol{g} \\
\bar{\rho}(\chi, t) \frac{\partial H(\chi, t)}{\partial t}-\bar{\rho}(\chi, t) \boldsymbol{u}_{\mathrm{mesh}} \cdot \nabla H(\chi, t)+\rho_{1} u(\chi, t) \cdot \nabla H_{1}(\chi, t) \\
=\nabla \cdot[k \nabla T(\chi, t)]+\left[H(\chi, t)-H_{1}(\chi, t)\right] \rho_{1} \nabla \cdot \boldsymbol{u}(\chi, t) \\
\bar{\rho}(\chi, t) \frac{\partial w(\chi, t)}{\partial t}-\bar{\rho}(\chi, t) \boldsymbol{u}_{\mathrm{mesh}} \cdot \nabla w(\chi, t)+\rho_{1} \boldsymbol{u}(\chi, t) \cdot \nabla w_{1}(\chi, t) \\
=\rho_{1} D_{1} \nabla \cdot\left[g_{1} \nabla w_{1}(\chi, t)\right]+\left[w(\chi, t)-w_{1}(\chi, t)\right] \rho_{1} \nabla \cdot \boldsymbol{u}(\chi, t)
\end{gathered}
$$

where $\chi$ denotes the ALE spatial system.

In the ALE formulations [Eqs. (7-10)], the variables are defined in the ALE spatial system $\chi$. The mesh is updated at each time step to account for the domain change caused by solidification shrinkage.

$$
\boldsymbol{X}(\chi, t+\Delta t)=X(\chi, t)+\boldsymbol{u}_{\text {mesh }}(\chi, t) \cdot \Delta t
$$

where $\boldsymbol{X}$ denotes the global vector of nodal coordinates, $\Delta t$ denotes the time step. The mesh velocity, $\boldsymbol{u}_{\text {mesh }}$, is determined by the mesh update algorithm, which has been introduced in a previous work $^{[7]}$.

\subsection{Numerical algorithm}

The finite element method is adopted to solve the conservation equations of the ALE model. The main solution algorithms are as follows ${ }^{[7]}$ :

(1) To deal with the convection induced instability, the Streamline-Upwind/Petrov-Galerkin (SUPG) stabilized finite element algorithm is adopted.

(2) Since the mass conservation equation is nonhomogeneous due to the existence of the solidification shrinkage source term, a modified pressure correction method is proposed to solve the velocity-pressure coupling between the mass and momentum conservation equations.

(3) As the ALE description is used, the motion of the mesh should be described. A mesh update algorithm based on Laplacian smoothing is developed to consider the domain change caused by solidification shrinkage.

(4) The assumption of the movement of the free surface is as follows: For nodes at the free surface, if the solid fraction is greater than the critical solid fraction, $g_{\text {sc }}$, then these nodes stay stationary. A default value of 0.99 is assumed for $g_{\text {sc }}$. For the other part of the free surface, which is usually at the middle of the free surface, the surface tension is neglected and the melt is assumed to go down with the same velocity, i.e. the feeding velocity.

(5) To solve the energy conservation equation, two numerical schemes are developed: the enthalpy-based approach and temperature-based approach. For the enthalpy-based approach, Eq. (9) is solved with the enthalpy as the solution variable. For the temperature-based approach, the temperature form of the energy conservation equation is utilized and the temperature is taken as the solution variable. Here, for the sake of simplicity, the heat capacities of the solid and liquid phases are assumed to be equal. Thus the temperature form of the energy conservation equation is as shown in Eq. (12). The Newton-Rapson method is adopted to solve Eq. (12), and the solid fraction is updated during the nonlinear iterations.

$$
\begin{aligned}
& \bar{\rho}(\chi, t) c_{\mathrm{p}} \frac{\partial T(\chi, t)}{\partial t}-\bar{\rho}(\chi, t) c_{\mathrm{p}} \boldsymbol{u}_{\mathrm{mesh}} \cdot \nabla T+\rho_{1} c_{\mathrm{p}} \boldsymbol{u}(\chi, t) \cdot \nabla T(\chi, t) \\
& =\nabla \cdot[k \nabla T(\chi, t)]+\rho_{\mathrm{s}} L\left(\frac{\partial g_{\mathrm{s}}}{\partial t}-\boldsymbol{u}_{\mathrm{mesh}} \cdot \nabla g_{\mathrm{s}}\right)
\end{aligned}
$$

where $c_{\mathrm{p}}$ is the specific heat and $g_{\mathrm{s}}$ is the local solid volume fraction.

(6) The solidification progress is assumed to be controlled by the lever rule and the phase diagram of the binary alloy. Thus, the mass fraction of liquid $f_{1}$ is calculated by solving Eqs. (13-15).

$$
\begin{aligned}
& w_{\mathrm{s}}=k_{\mathrm{p}} w_{1} \\
& w=f_{\mathrm{s}} w_{\mathrm{s}}+f_{1} w_{1} \\
& T=T_{\mathrm{f}}+m w_{1}
\end{aligned}
$$

where $k_{\mathrm{p}}$ is the partition coefficient, $m$ is the liquidus slope, $T_{\mathrm{f}}$ is the pure metal melting point. Then, the volume fraction of liquid $g_{1}$ can be updated by Eq. (16).

$$
g_{1}=\frac{f_{1} / \rho_{1}}{f_{\mathrm{s}} / \rho_{\mathrm{s}}+f_{1} / \rho_{1}}
$$




\section{Validation}

The widely-used Hebditch-Hunt benchmark ${ }^{[19,20]}$ is used to validate the newly-developed volume-averaged finite element model and the temperature-based energy equation solver for macrosegregation. This benchmark is a solidifying $\mathrm{Pb}-48 \mathrm{wt}$ \% $\mathrm{Sn}$ alloy ingot in a parallelepipedic cavity which is $100 \mathrm{~mm}$ long, $60 \mathrm{~mm}$ high and $13 \mathrm{~mm}$ wide. Here, solidification shrinkage is neglected, thus the ALE model degenerates to the traditional fixed-domain model. The schematic, computational parameters and other details about the benchmark can be found in the references ${ }^{[20,21]}$. The segregation ratios along four horizontal section lines at different heights are shown in Fig. 1. The predictions by the temperature-based energy solver agree well with those by the enthalpy-based energy solver, and they all agree well with the measurements. Thus, the accuracy of the temperature-based energy solver can be approved and the volume-averaged finite element macrosegregation model can be validated. For the convenience of applying Dirichlet boundary conditions, the temperaturebased energy solver is used in the following study.

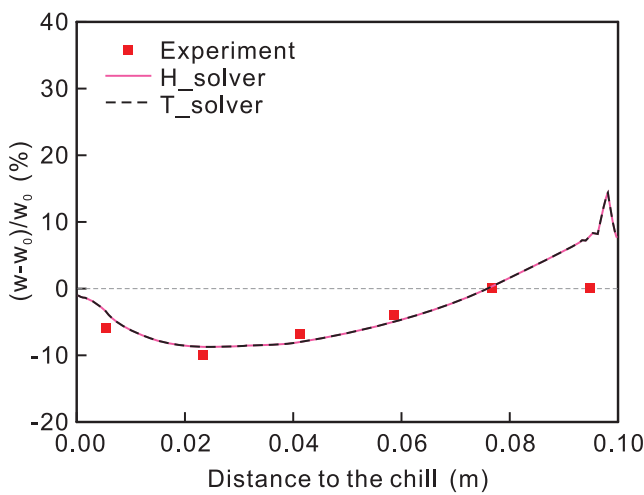

(b) $y=25 \mathrm{~mm}$

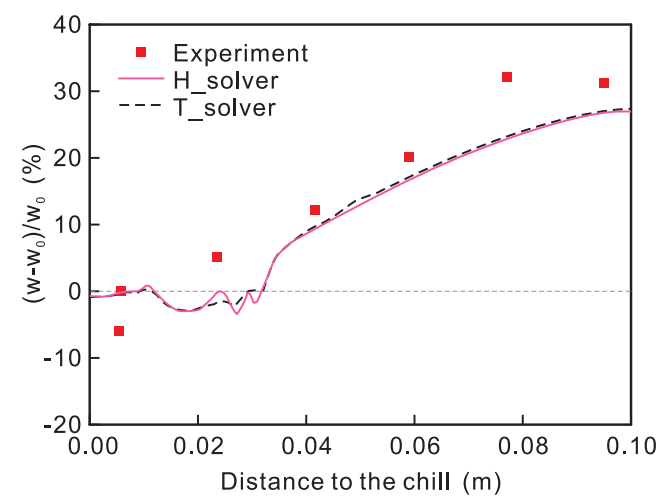

(d) $y=55 \mathrm{~mm}$

Fig. 1: Final macrosegregation profiles along four cross section lines at different heights of the ingot

\section{Results and discussion}

\subsection{Steel ingot description}

To verify the ALE model, it was used to predict macrosegregation coupled with shrinkage cavity in a 3.3 industrial steel ingot, produced by the company Aubert \& Duval ${ }^{[22]}$. Some simplifications regarding the ingot configuration and heat exchange conditions were adopted for the application of a two-phase finite element model ${ }^{[23]}$. The steel was simplified as a $\mathrm{Fe}-0.36 \mathrm{wt} . \% \mathrm{C}$ binary alloy and the ingot configuration was simplified as cylindrical. The top surface was assumed adiabatic due to the existence of the exothermal powder layer. The heat exchanges through the mould and the refractory were modelled by applying Fourier type boundary conditions to the lateral and bottom surfaces. Two different convective heat transfer coefficients were used: $h_{1}=1000 \mathrm{~W} \cdot \mathrm{m}^{-2} \cdot{ }^{\circ} \mathrm{C}^{-1}$, in both the lower zone of the lateral surface (1.4 m height from the bottom) and the bottom surface, representing faster cooling via the grey iron mould; and $h_{2}=700 \mathrm{~W} \cdot \mathrm{m}^{-2} \cdot{ }^{\circ} \mathrm{C}^{-1}$ in the upper zone ( $0.4 \mathrm{~m}$ depth from the top) representing a limited heat exaction through refractory. Due to the symmetry of the ingot system, a half of the ingot system is used for simulation. The schematic of the $3.3 \mathrm{t}$ steel ingot system with thermal boundary conditions is shown in Fig. 2. The related computation parameters ${ }^{[22-23]}$ are given in Table 1.

In this study, besides the simulation by the ALE model, two other simulations by a finite element model and a finite volume model ${ }^{[21]}$ are conducted. The finite element model is a degenerated version of the ALE model by neglecting solidification shrinkage and the mesh movement. The densities of the solid and liquid phases are assumed to be equal, thus only the thermal-solutal convection factor is considered for 


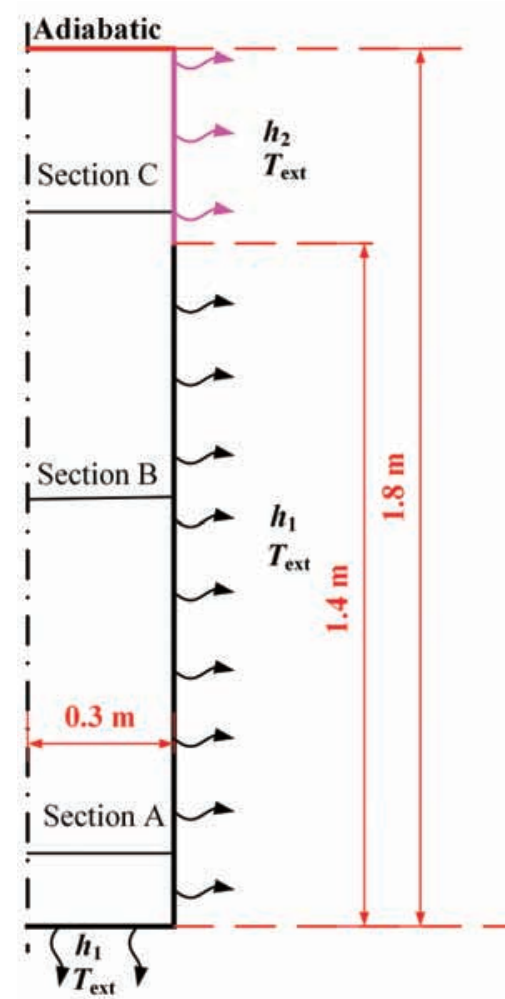

Fig. 2: Schematic of $3.3 \mathrm{t}$ steel ingot system with thermal boundary conditions
Table 1: Thermo-physical properties and computational parameters of $3.3 \mathrm{t}$ steel ingot case ${ }^{[22-23]}$

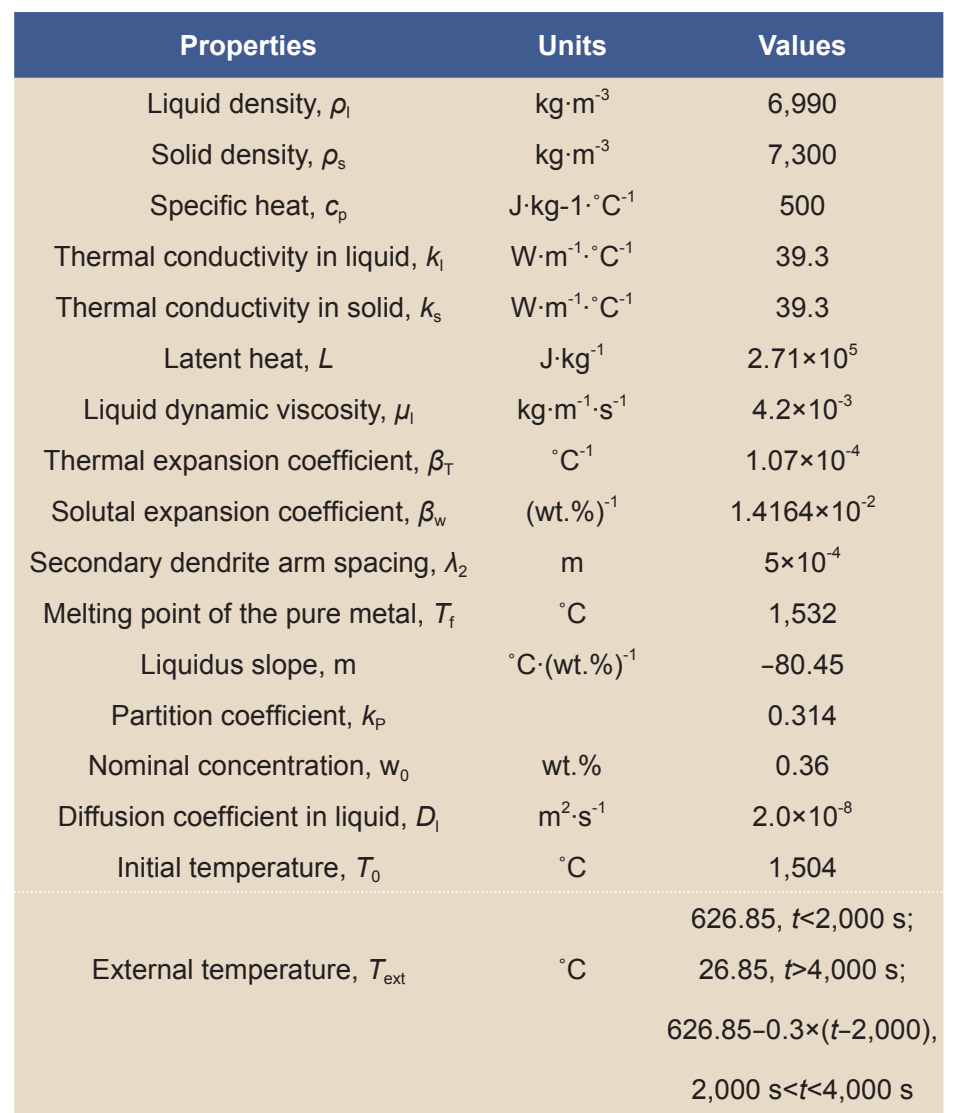

macrosegregation formation. The finite volume model solves the same governing equations as the finite element model by using the finite volume method. In all the three simulations, the same computational parameters are used. By convergence studies of the mesh size and time step, a structured mesh with a size of $5 \mathrm{~mm}$ and a time-step of $0.05 \mathrm{~s}$ is adopted for the three simulations. In the following sections, the solidification process predicted by the ALE model is analyzed at first, then predictions by three models are compared and the influence of solidification shrinkage is investigated.

\subsection{Solidification process and macrosegregation formation}

Figure 3 shows the evolutions of the temperature field and solid fraction distribution predicted by the ALE model. The left panels show the temperature fields and the right panels indicate the solid fraction. The mushy zones are thin due to the narrow solidification temperature range. The solidification progress is mainly governed by the heat extraction condition. Due to the cooling of the mold and the insulation of the adiabatic surface, solidification starts from the bottom and the lateral regions of the ingot and a " $U$ " shaped liquid region forms in the ingot. At the beginning of solidification, the contours of the temperature field and the solid fraction are generally straight, and only bend at the upper zone of the ingot due to the insulation effect of the refractory sleeve, as shown in Fig. 3(a). As solidification progresses, the temperature continues to decrease, and the " $U$ " shaped liquid region gradually turns into a narrow channel at

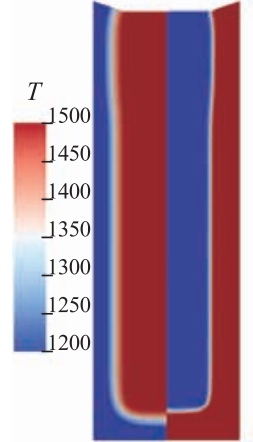

(a)

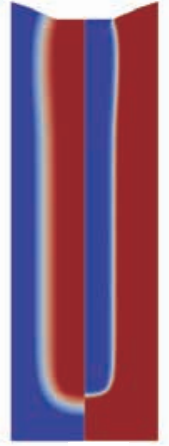

(b)

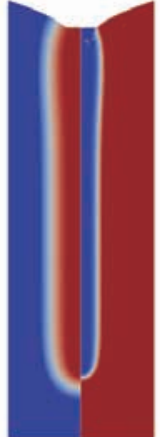

(c)

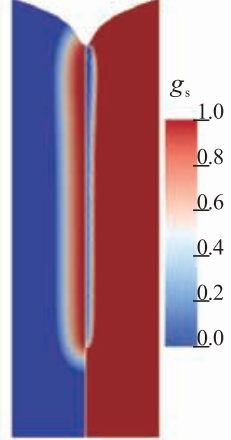

(d)
Fig. 3: Evolutions of temperature field (left) and solid fraction (right) in the ingot at different times: (a) $t=1,000 \mathrm{~s}$, (b) $t=2,000 \mathrm{~s}$, (c) $t=3,000 \mathrm{~s}$ and (d) $t=4,000 \mathrm{~s}$

the center of the ingot, as shown in Figs. 3(b), 3(c) and 3(d). Besides, due to solidification shrinkage, the melt in the upper zone of the ingot flows downwards to compensate the volume shrinkage. The ingot surface declines and a shrinkage cavity forms gradually, as indicated in Fig. 3.

Figure 4 shows the evolutions of the velocity field and solute concentration predicted by the ALE model. The left panels show the solid fraction and the average velocity fields, while the right panels show the solute concentration fields with two white lines indicating the solid fraction of 0.1 and 0.9 . In this study, the solid motion is neglected, thus the fluid flow is driven only by the combined effect of thermal-solutal buoyancy force and solidification shrinkage, causing the redistribution 


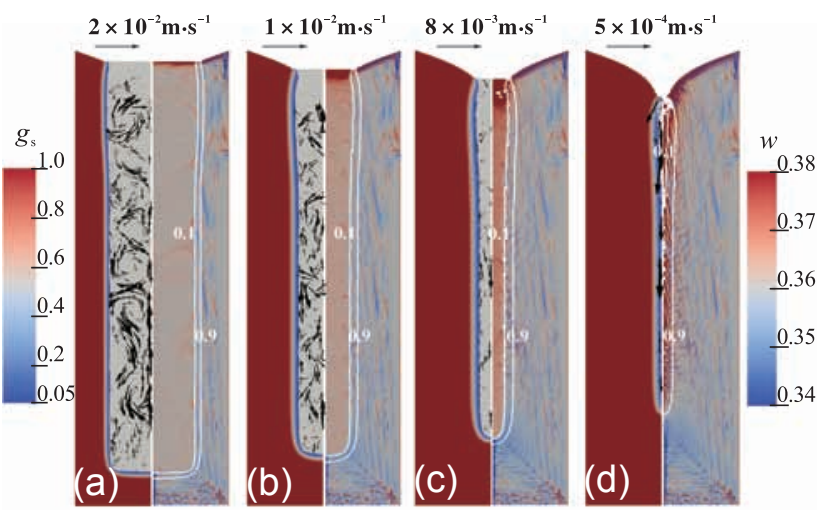

Fig. 4: Evolutions of velocity field (left) and solute concentration (right) in the ingot at different times: (a) $t=1,000 \mathrm{~s}$, (b) $t=2,000 \mathrm{~s}$, (c) $t=3,000 \mathrm{~s}$ and (c) $t=4,000 \mathrm{~s}$

of solute concentration. At the early stage of solidification, the effect of thermal-solutal buoyancy force dominates over the shrinkage effect, and the flow is rather complicated with some vortexes, as shown in Fig. 4(a). As solidification progresses, the liquid region gradually decreases and the flow weakens, as indicated by the decrease of the velocity magnitude in Figs. 4(b), 4(c) and 4(d). In this fixed solid simulation case, the development of the segregation depends on the direction of the temperature gradient and the liquid flow. The negative segregation cone forms at the bottom at first and continues to develop, while the liquid region in the center is more and more enriched, thus forming positive segregation above the negative segregation cone. Besides, strong and banded A-segregates form at most part of the ingot body. These segregates develop due to the instabilities in the mushy zone that perturbs the fluid flow at the scale of a few centimeters. In this study, the mesh with a grid size of $5 \mathrm{~mm}$ allows the capture of this mesoscale structure.

\subsection{Comparison of different models and influence of shrinkage cavity formation}

Figure 5 shows the macrosegregation maps at the end of solidification predicted by the three models: (a) ALE model, (b) finite element model and (c) finite volume model. As stated earlier, the same computational parameters and mesh are used
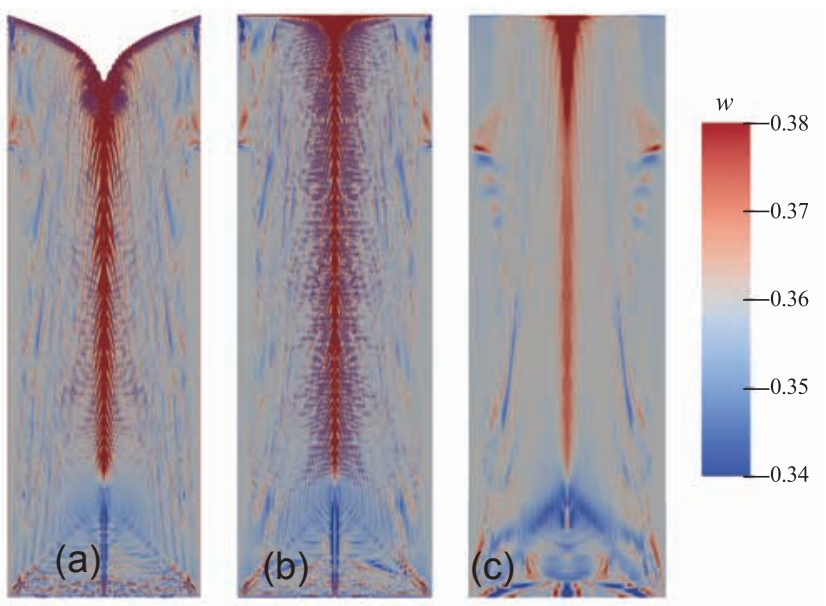

Fig. 5: Final macrosegregation maps: (a) ALE model, (b) finite element model and (c) finite volume model for the three models. Generally, similar macrosegregation patterns are predicted, and the commonalities are as follows: Firstly, a negative segregation zone exists in the bottom of the ingot while a narrow positive segregation channel region forms from the top surface deep into the ingot along the centreline. Secondly, the strong and banded A-segregates are predicted at most regions of the ingot. Thirdly, adjacent positive segregation and negative segregation form at the height of $1.4 \mathrm{~m}$ of the steel ingot while no obvious segregation forms at the other part of the lateral surface. Due to the negligence of grain sedimentation, which is an important factor for the formation of the conic negative segregation at the bottom of ingot, the formation of negative segregation at the bottom and the positive segregation at the top are mainly caused by the solutal buoyancy. As pointed out previously, the narrow deep liquid region channel at the later stage of solidification in Fig. 3(d) is enriched in solute and the liquid flow is very weak, thus the narrow positive segregation region forms in the centre at the end of solidification. In summary, the macrosegregation maps predicted by the three models generally agree with each other, thus it can prove calculation accuracy of the three models. However, some differences exist between predictions by the three models, which result from the different mechanisms and numerical methods.

Figures 5(a) and 5(b) show the predictions by using the finite element method while Fig. 5(c) shows the prediction by using the finite volume method. Comparing Fig. 5(b) with Fig. 5(c), the main difference is that A-segregates predicted by the finite element method are stronger and more developed than those predicted by finite volume method. For the prediction using the finite element method, A-segregates occupy most part of the ingot body, and many small channels exist just adjacent to the center positive region. However, for the prediction using the finite volume method, obvious segregation channels only exist near the half radius region of the ingot. Since the model equations are the same for Fig. 5(b) and Fig. 5(c), the reasons for the differences may be the inherent difference between the finite element method and the finite volume method and the different convection stabilization techniques.

From the perspective of the macrosegregation formation mechanism, the ALE model [Fig. 5(a)] considers the combined effect of thermal-solutal convection and solidification shrinkage, while the finite element model [Fig. 5(b)] and finite volume model [Fig. 5(c)] only consider the effect of thermal-solutal convection. To quantitatively investigate the influence of solidification shrinkage on macrosegregation formation, the macrosegregation ratios along the centerline and three cross section lines at different heights of the ingot, section A, section $\mathrm{B}$ and section C, as indicated in Fig. 2, are shown in Fig. 6. FEM and FVM in Fig. 6 represent the finite element model and finite volume model. Firstly, compared with the case without solidification shrinkage [Fig. 6(b)], the positive segregation zone at the hot top is in a lower place [Fig. 6(a)]. The reason seems obvious, since the formation of shrinkage cavity will inevitably adjust the positive segregation region at the upper zone of the ingot to a lower position. Secondly, compared 

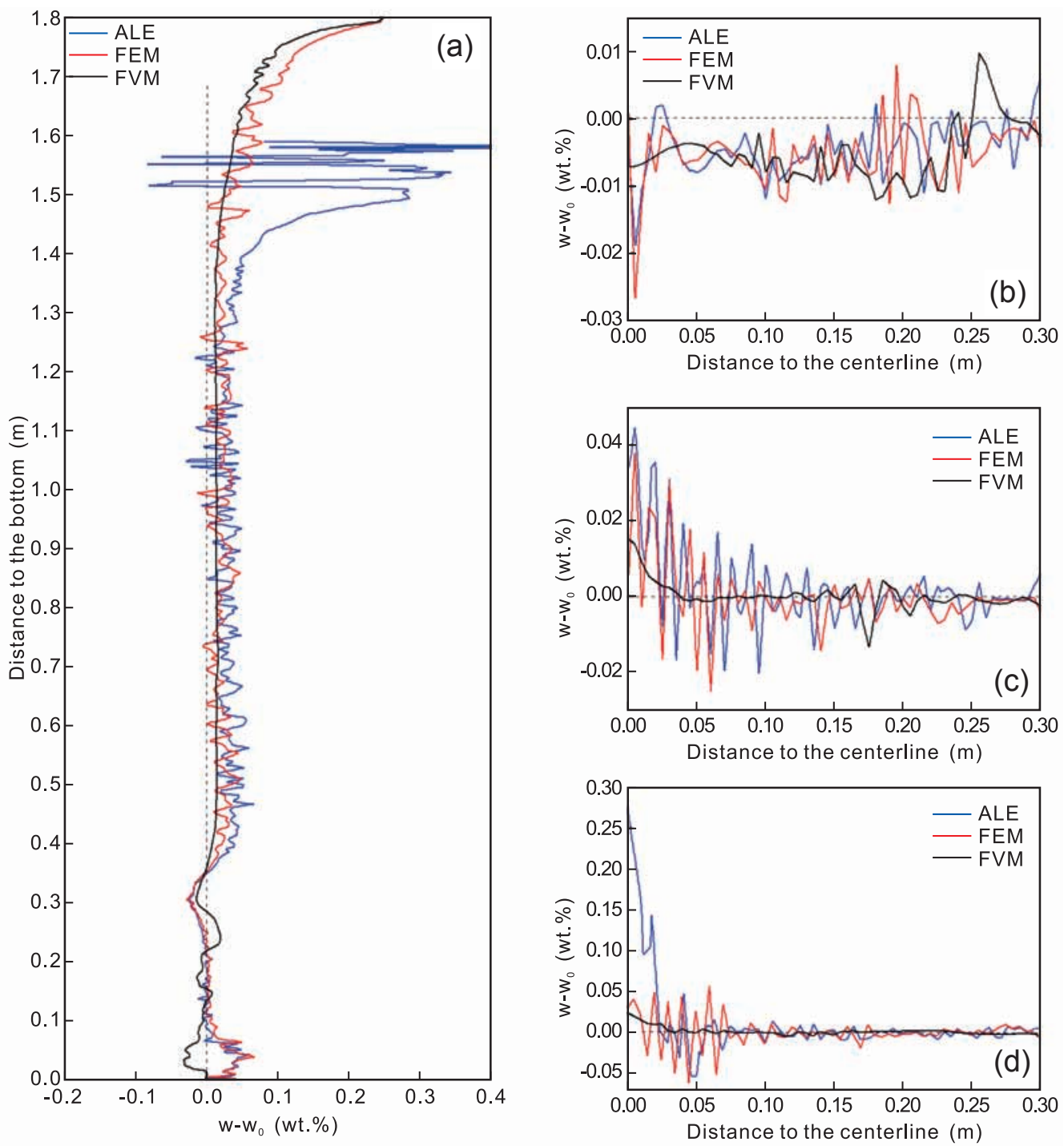

Fig. 6: Macrosegregation ratios along the centerline (a) and three cross section lines of ingot: (b) section $A$, (c) section $B$ and (d) section C

to the case without solidification shrinkage, the positive segregation at the top of the centerline is more severe with solidification shrinkage, as shown in Fig. 6(a). Thirdly, when the solidification shrinkage is considered, the severity of the negative segregation band near the ingot wall is reduced. The negative segregation at the wall turns into the weak positive segregation, as shown in Figs. 6(b), 6(c) and 6(d), which corresponds to the formation of reverse segregation.

To sum up, the positive segregation at the hot top is underestimated by the finite element model and finite volume model neglecting solidification shrinkage. The ALE model provides a more accurate prediction by considering shrinkage cavity. The effects of the formation of shrinkage cavity on macrosegregation are mainly in the following aspects: (1) for the positive segregation at the hot top, the shrinkage not only adjusts the positive segregation zone to a lower position, but also increases the severity of the positive zone; (2) the solidification shrinkage is responsible for the inverse segregation at the wall and the reduction of the degree of the negative segregation band near the wall.

\subsection{Sensitivity study of the critical solid fraction $g_{\mathrm{sc}}$}

In the mesh update algorithm of the ALE model, a critical solid volume fraction, $g_{\text {sc }}$, is designed to determine the motion state of the nodes at the free surface (the top surface of the ingot in this case). When the local solid volume fraction is larger than the critical solid volume fraction $\left(g_{\mathrm{s}}>g_{\mathrm{sc}}\right)$, the node stays stationary. In the previous simulation, the critical solid volume fraction is set to 0.99 , which means that except for the nodes in pure solid state, the other nodes in mushy state or pure liquid state at the free surface can move downwards.

In order to investigate the influence of the critical solid volume fraction, $g_{\text {sc }}$, on macrosegregation predicted using the ALE model, two cases are added: $g_{\mathrm{sc}}=0.7$ and $g_{\mathrm{sc}}=0.3$. Figure 7 shows the final macrosegregation maps predicted using the ALE model with different critical solid volume fractions. In general, the final macrosegregation maps predicted with three critical solid volume fractions are similar, including negative segregation at the bottom, positive segregation in the center core and banded A segregates at the centerline. However, 

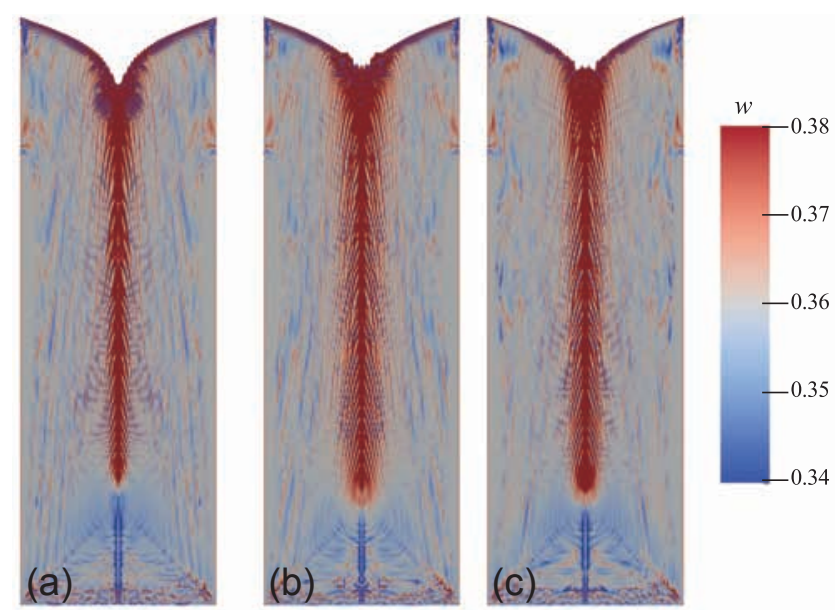

Fig. 7: Final macrosegregation maps predicted using ALE model with different critical solid volume fractions: (a) 0.99, (b) 0.7 and (c) 0.3

some small differences exist with regard to the shape of shrinkage cavity and positive segregation along the centerline. First, with the decrease of the critical solid volume fraction, the area of positive segregation at the center of the ingot increases slightly. Secondly, as the critical solid volume fraction decreases, the outer part of the shrinkage cavity is not much different. But, for the center part of the shrinkage cavity, the cavity shape becomes flatter. For $g_{\text {sc }}=$ 0.99 , the center part of the shrinkage cavity is V-shaped; while for $g_{\mathrm{sc}}=0.3$, it turns into U-shaped.

The differences in predictions using different critical solid volume fractions can be explained as follows: In the mesh update algorithm, the critical solid volume fraction is set to determine the number of the moving nodes at the free surface. As the critical solid volume fraction decreases, the number of the moving nodes reduces; thus the area of the downward moving surface reduces, and the feeding velocity increases. At the late stage of solidification, the nodes at the central region of the free surface are all in mushy state. For $g_{\mathrm{sc}}=0.3$, the solid volume fractions of nodes at the free surface may be all larger than $g_{\text {sc }}$, then all the nodes in the free surface stop moving downwards to compensate for the solidification shrinkage, the center part of the shrinkage cavity presents $U$ type. For $g_{\text {sc }}=0.99$, nodes in mushy state at the free surface can still move downwards, thus, the shrinkage cavity is shown as V-shaped in the central region.

\section{Conclusions}

In this paper, an ALE model considering the combined effect of solidification shrinkage and thermal-solutal convection is utilized to predict the formation of macrosegregation and shrinkage cavity during solidification of an industrial steel ingot. Different from the previous fixed-domain models used for macrosegregation, the formation of shrinkage cavity is solved by a moving mesh method. The volume averaging method is adopted to derive the model. After validation by a Pb-48wt.\%Sn solidification benchmark, the ALE model is applied to a $3.3 \mathrm{t}$ steel ingot to test the ability for coupling the prediction of macrosegregation and shrinkage cavity. The following conclusions are drawn:

(1) The ALE model can be used to predict macrosegregation and shrinkage cavity for steel ingots.

(2) The formation of shrinkage cavity influences the macrosegregation map, thus it is necessary to consider the shrinkage cavity formation in macrosegregation models to achieve better agreement.

(3) Solidification shrinkage is responsible for the inverse segregation at the wall and adjusts the position of the positive segregation zone at the hot top of the ingot. For better application of the ALE model, grain sedimentation should be further included and further experiment verifications in terms of macrosegregation and shrinkage cavity are required.

\section{References}

[1] Flemings M C. Our understanding of macrosegregation: past and present. ISIJ International, 2000, 40(9): 833-841.

[2] Beckermann C. Modelling of macrosegregation: applications and future needs. International Materials Reviews, 2002, 47(5): 243-261.

[3] Ma X P, Li D Z. Macrosegregation and its formation mechanism in steel ingot with designed local thermal control. ISIJ International, 2014, 54(2): 356-358.

[4] Krane M J M, Incropera F P. Analysis of the effect of shrinkage on macrosegregation in alloy solidification. Metallurgical and Materials Transactions A, 1995, 26(9): 2329-2339.

[5] Heinrich J C, Poirier D R. The effect of volume change during directional solidification of binary alloys. Modelling and Simulation in Materials Science and Engineering, 2004, 12(5): 881-899.

[6] Samanta D, Zabaras N. Modelling convection in solidification processes using stabilized finite element techniques. International Journal for Numerical Methods in Engineering, 2005, 64(13): 1769-1799.

[7] Chen KX, Shen H F. Numerical simulation of macrosegregation caused by thermal-solutal convection and solidification shrinkage using ALE model. Acta Metallurgica Sinica (English Letters), 2019, https://doi.org/10.1007/s40195-019-00897-0.

[8] Zhang S L, Yanke J, R. Johnson D R, et al. Modeling defects in castings using a volume of fluid method. International Journal of Numerical Methods for Heat \& Fluid Flow, 2014, 24(2): 468-482.

[9] Zhang S L, Johnson D R, Krane M J M. Influence of riser design on macrosegregation in static castings. International Journal of Cast Metals Research, 2015, 28(1): 28-38.

[10] Wang T M, Yao S, Zhang X G, et al. Modelling of the thermosolutal convection, shrinkage flow and grain movement during globular equiaxed solidification in a multi-phase system - I. Three-phase flow model. Acta Metallurgica Sinica, 2006, 42(6): 584-590. (In Chinese)

[11] Wang T M, Li T J, Cao Z Q, et al. Modelling of the thermosolutal convection, shrinkage flow and grain movement during globular equiaxed solidification in a multi-phase system-II. Application of model. Acta Metallurgica Sinica, 2006, 42(6): 591-598. (In Chinese)

[12] Wu M, Kharicha A, Ludwig A. Using four-phase Eulerian volume averaging approach to model macrosegregation and shrinkage cavity. In: IOP Conference Series: Materials Science and Engineering, IOP Publishing, 2015: 012006.

[13] Wu M, Ludwig A, Kharicha A. A four phase model for the macrosegregation and shrinkage cavity during solidification of steel ingot. Applied Mathematical Modelling, 2017, 41: 102-120. 
[14] Ge H H, Ren F L, Li J, et al. Four-phase dendritic model for the prediction of macrosegregation, shrinkage cavity, and porosity in a 55-ton ingot. Metallurgical and Materials Transactions A, 2017, 48(3): 1-12.

[15] Ren F L, Ge H H, Cai D X, et al. Simulation of macrosegregation and shrinkage cavity in an $\mathrm{Al}-4.5$ wt pct $\mathrm{Cu}$ ingot using a fourphase model. Metallurgical and Materials Transactions A, 2018(12): 6243-6254.

[16] Bellet M, Fachinotti V D. ALE method for solidification modelling. Computer Methods in Applied Mechanics and Engineering, 2004, 193(39-41): 4355-4381.

[17] Bellet M, Jaouen O, Poitrault I. An ALE-FEM approach to the thermomechanics of solidification processes with application to the prediction of pipe shrinkage. International Journal of Numerical Methods for Heat and Fluid Flow, 2005, 15(2): 120-142.

[18] Belytschko T. Liu W K. Moran B. Nonlinear finite elements for continua and structures. 2nd ed., West Sussex, UK, John Wiley \& Sons Inc., 2014: 417-475.

[19] Hebditch D J, Hunt J D. Observations of ingot macrosegregation on model systems. Metallurgical Transactions, 1974, 5(7): 1557-1564.
[20] Ahmad N, Rappaz J, Desbiolles J L, et al. Numerical simulation of macrosegregation: a comparison between finite volume method and finite element method predictions and a confrontation with experiments. Metallurgical and Materials Transactions A, 1998, 29(2): 617-630.

[21] Li W S, Shen H F, Liu B C. A response to numerical benchmark for solidification of binary alloys:macro-segregation with thermosolutal convection. China Foundry, 2012, 9(2): 171177.

[22] Combeau H, Založnik M, Hans S, et al. Prediction of macrosegregation in steel ingots: influence of the motion and the morphology of equiaxed grains. Metallurgical and Materials Transactions B, 2009, 40(3): 289-304.

[23] Nguyen T T M, Gandin C A, Combeau H, et al. Finite element multi-scale modeling of chemical segregation in steel solidification taking into account the transport of equiaxed grains. Metallurgical and Materials Transactions A, 2018, 49(5): 1725-1748. 